\title{
Complicaciones de personas adultas con cáncer gástrico tratadas por gastrectomía. Una revisión de literatura
}

\section{The complications of adults with gastric cancer who treated by gastrectomy. A literature review}

\author{
Luz Amparo Leiva-Morales (iD) 1 \\ 1. Universidad Nacional de Colombia. Bogotá, Colombia. Correo: laleivam@unal.edu.co - https://orcid.org/0000-0002-7777-1116 \\ Tipología: Artículo de revisión \\ Para citar este artículo: Leiva-Morales, L. Complicaciones de personas adultas con cáncer gástrico tratadas por gastrectomía. Una revisión de literatura. \\ Duazary. 2021 abril; 18(2): 199-212. Doi: https://doi.org/10.21676/2389783X.4072
}

\section{RESUMEN}

Palabras clave: complicaciones posoperatorias; adulto; gastrectomía; neoplasias gástricas.
Enfermería, como parte de un equipo interdisciplinario, requiere identificar las complicaciones de una gastrectomía para proporcionar un cuidado integral al paciente. La clasificación Clavien-Dindo es una herramienta que facilita este proceso. El objetivo de este artículo es describir las complicaciones de personas adultas con cáncer gástrico tratadas por gastrectomía a partir de una revisión de literatura, mediante búsqueda de artículos en cinco bases de datos indexadas. Se incluyeron artículos de texto completo, en idiomas inglés, español y portugués, de los años 2008-2018, con palabras claves de acuerdo a los tesauros validados en DECS y MESH. Se construyeron ecuaciones de búsqueda, una matriz de análisis y la clasificación Clavien-Dindo. En general, 27 estudios cumplieron con los criterios de inclusión, $100 \%$ cuantitativos. Las complicaciones se relacionan con aspectos físicos $\mathrm{y} / \mathrm{o}$ emocionales preoperatorios que conllevan a los pacientes a: infección órgano-espacio $(9,1 \%)$; absceso intraabdominal $(6,6 \%)$; infección de la herida o seroma (5,6\%); fugas anastomóticas (4,5\%); molestias gastrointestinales $(3,3 \%)$; agotamiento $(3,1 \%)$ y alteración laboral $(3,1 \%)$. Esto permite concluir que el preoperatorio cumple un papel preponderante en el proceso quirúrgico porque incide en las complicaciones posoperatorias, especialmente en personas que tengan alteraciones nutricionales, ya sea por exceso como por déficit.

\section{ABSTRACT}

\section{Keywords:}

Post-

operative complications; Adult; Gastrectomy; Gastric neoplasia.
Nursing as part of the interdisciplinary equipement requires identifying the complications of a gastrectomy to provide comprehensive care to the patient, the Clavien-Dindo classification is a tool that facilitates this process. The objective of this article is to describe complications of adults with gastric cancer who underwent a gastrectomy from a systematic review of literature. by searching of articles in five indexed data bases. There were included articles full text, English, Spanish and Portuguese languages, years 2008-2018, key words according to thesaurus validated in DECS y MESH, there were built search equations, analysis matrix and Clavien-Dindo classification. Overall, 27 studies accomplished the inclusion criteria, $100 \%$ quantitative. The more reported complications in the documents are related with physical and/or emotional aspects like: infection organ space (9.1\%); intraabdominal abscess (6.6\%), wound infection or seroma (5.6\%) anastomotic leaks (4.5\%); gastrointestinal pain (3.3\%); exhaustion (3.1\%) and working alteration (3.1\%). What allows us to conclude is that preoperative plays a prevailing function in the surgical process because it has an impact on the post-operative complications, especially in patients that have nutritional alterations, either by excess or by deficit. 


\section{INTRODUCCIÓN}

En 2013 se presentaron 984.000 casos nuevos de cáncer gástrico en el mundo, de los cuales 841.000 terminaron en fallecimiento. De esta forma, llegó a representar la segunda causa de muerte por cáncer y la quinta en incidencia anual por tumores malignos ${ }^{1-3}$. Según la Organización de Naciones Unidas (ONU), uno de cada 36 hombres y una de cada 84 mujeres desarrollará cáncer gástrico antes de los 79 años, siendo prevalente esta enfermedad en América Latina, Asia del Este, áreas de Europa y Medio Este. Sin embargo, el $60 \%$ del total de cánceres gástricos en el mundo están concentrados en Japón, China y Corea ${ }^{4}$.

El cáncer gástrico es una enfermedad con múltiples agentes causales ${ }^{5}$ tales como factores genéticos o ambientales -asociados con infecciones por Helicobacter pylori, el cual se ha identificado como carcinógeno en $60-70 \%$ de los casos $-{ }^{6}$. Los países occidentales han informado tasas de morbimortalidad de $35-46 \%$ y de $4-16 \%$ respectivamente. Después de la disección de los ganglios linfáticos, las complicaciones incluyen fuga anastomótica, hemorragia y absceso intraabdominal, obstrucción intestinal, pancreatitis y dehiscencia de la herida. La reoperación de estas complicaciones varía de $2,8 \%$ a $10 \%{ }^{7}$.

En Colombia, la incidencia de cáncer es de 62.818 casos entre 2007-2011: 47,3\% en hombres, en quienes prevalecen los cánceres de próstata, estómago y pulmón, entre otros, y el $52,6 \%$ en mujeres, especialmente el de mama, el de cérvix, el del colon, entre otros ${ }^{8}$. En el 2015 el cáncer gástrico afectó a 6028 pacientes en el país, con edad promedio de 62,8 años, y el $90 \%$ de los casos se dio en mayores de 45 años, con prevalencia en hombres. La mortalidad de este tipo de cáncer es de 1696, es decir, 3,5 por 100.000 habitantes $^{9}$.

El tratamiento del cáncer gástrico es fundamentalmente quirúrgico (gastrectomía), y consiste en la resección del tumor y de los tejidos circundantes y linfáticos. De acuerdo con un estudio, la supervivencia posquirúrgica a cinco años en estos casos solo alcanza un $10 \%$ a $15 \%{ }^{10}$, aunque en
Colombia se ha demostrado que puede ser del $54 \%{ }^{11}$. La gastrectomía se puede realizar por dos vías: clásica (abierta) o laparoscópica (cerrada). Esta última favorece la disección ganglionar por la excelente exposición retroperitoneal que se obtiene ${ }^{12,13}$. Dependiendo de la ubicación donde se encuentre el cáncer, se clasifica en cirugía total y subtotal. La primera se emplea si el cáncer se ha propagado por todo el estómago, o si el cáncer se encuentra en la parte superior del estómago, cerca del esófago, en cuyo caso el tratamiento consiste en realizar anastomosis del intestino delgado, facilitando el paso de los alimentos, motivo por el cual se recomienda administrar una dieta fraccionada ${ }^{12}$. La segunda se utiliza si el cáncer está ubicado únicamente en la parte inferior o superior del estómago. En estos casos se extirpa una parte de él o la primera parte del intestino delgado, reconectando la parte restante del estómago. Este tratamiento quirúrgico permite mejorar el estado nutricional y, por consiguiente, tener calidad de vida $^{12}$

De acuerdo con la literatura, la gastrectomía se considera una cirugía compleja por las complicaciones que se presentan en el posoperatorio $^{12}$. Según el Instituto Nacional de Cancerología, "después de una gastrectomía total o subtotal, no se le permitirá ingerir alimentos o bebidas al menos por varios días. Esto se hace para permitir que el tracto digestivo sane y para asegurar que no existen fugas en partes que han sido suturadas durante la operación" ${ }^{12}$. Sin embargo, podrían presentarse efectos secundarios durante la recuperación, principalmente posteriores a la ingesta de alimentos, como epigastralgia, dolor abdominal y diarrea. Este proceso se da por la extirpación que ha sufrido el estómago, lo que hace que los alimentos transiten con mayor rapidez por el tracto digestivo. De acuerdo a la literatura, los efectos adversos de la gastrectomía duran más tiempo que en otros tipos de cirugía, y la readmisión temprana podría darse seis meses después de la operación ${ }^{14}$. No obstante, no hay una definición estándar para estos eventos, ni del tiempo, ni de su gravedad, lo que dificulta la comparación precisa entre los estudios ${ }^{15}$. Ahora bien, la estandarización de las complicaciones posquirúrgicas mediante la 
clasificación Clavien-Dindo facilita la comparación de resultados de investigación ${ }^{16}$.

Cabe anotar que el personal de enfermería cumple un papel importante en el equipo multidisciplinario desde la prevención, el diagnóstico, el tratamiento y la recuperación del paciente ${ }^{17}$, incluyendo el egreso, por lo que se hace necesario que estos profesionales conozcan las posibles complicaciones de la cirugía para prevenir y actuar de manera oportuna brindando cuidados integrales al paciente. Pese a esto, en enfermería no se encuentran reportes en la literatura que den cuenta de la necesidad de conocer las complicaciones de una gastrectomía en adultos, con el fin de planear un cuidado integral a estas personas y sus familias. Por lo tanto, el objetivo del presente artículo es describir las complicaciones de personas adultas con cáncer gástrico tratadas por gastrectomía a partir de una revisión de literatura que permita reconocer las principales complicaciones que se pueden presentar durante el posoperatorio con el fin de que sea un recurso para generar intervenciones que mejoren la calidad de vida de estas personas.

\section{MATERIALES Y MÉTODOS}

\section{Tipo de estudio}

Revisión de literatura mediante la búsqueda de artículos en cinco bases de datos indexadas: PubMed, Science Direct, Biblioteca Virtual de Ciencias de la Salud, Google Academic y Ebsco.

\section{Criterios de inclusión}

Artículos con texto completo, en idiomas inglés, español y portugués, publicados entre los años 2008 y 2018 , que utilicen las palabras claves de acuerdo a los tesauros validados en DECS - "herida quirúrgica", "alta del paciente", "gastrectomía", "abdomen", "planificación de atención al paciente" y "adulto" - y en MESH - "surgical wound", "patient discharge", "gastrectomy", "abdomen", "care planning" y "adult" - con las que se construyeron seis ecuaciones de búsqueda, empleando los booleanos and y or.

\section{Criterios de exclusión}

a. Artículos que no se relacionaran con gastrectomía, o que estuvieran directamente relacionados con gastrectomía bariátrica; b. Costo para acceder; c. Publicaciones en idioma diferente al inglés, el español o el portugués.

\section{Instrumento}

STROBE es un instrumento aplicado para estudios de tipo observacional que está constituido por 22 ítems sobre el título de artículos, resumen, introducción, métodos, resultados, secciones de discusión y otras informaciones ${ }^{18}$.

\section{Procedimiento}

Para este proceso se construyó un formato de búsqueda de literatura donde se indicaba el número de artículos encontrados según la ecuación de búsqueda, la base de datos y la cantidad de artículos que, de acuerdo al título y al objetivo, estaban relacionados con el estudio planteado. La búsqueda se realizó durante cuatro meses (septiembrediciembre 2018). Posteriormente, se efectuó un segundo filtro de los artículos seleccionados donde se revisó el cuerpo del artículo.

Una vez hecho lo anterior, se procedió a aplicar el instrumento STROBE para estudios cuantitativos con el fin de obtener una revisión crítica (es de aclarar que los artículos cualitativos encontrados en la revisión no cumplieron con los criterios establecidos para el primer filtro de búsqueda). Para la segunda revisión la investigadora definió como criterio de inclusión que, al aplicarse el instrumento, los artículos obtuvieran una puntuación mayor o igual a $70 \%$ de acuerdo a los criterios del instrumento (Tabla 1).

Luego del proceso anterior, se construyó una matriz de análisis por base de datos donde se detallaban características específicas de los artículos como, por ejemplo, año, idioma, país, autores, institución donde se había desarrollado, objetivo del estudio, tipo de estudio, características sociodemográficas, tipo de cirugía y sus complicaciones. Estas particularidades fueron categorizadas de acuerdo a la clasificación Clavien-Dindo, aplicable a todo tipo 
de cirugía, bien sea abierta o por laparoscopia, que permite clasificar las complicaciones según el tipo de complejidad del tratamiento ${ }^{19,20}$.

Tabla 1. Resultados del proceso de búsqueda de literatura.

\begin{tabular}{|c|c|c|c|c|}
\hline Base de datos & $\begin{array}{c}\text { Resultados de } \\
\text { búsqueda }\end{array}$ & Primer filtro & $\begin{array}{c}\text { Aplicación del } \\
\text { instrumento }\end{array}$ & Elegidos \\
\hline PubMed & 13 & 4 & 4 & 4 \\
\hline Ebsco & 2491 & 28 & 0 & 0 \\
\hline Google Académico & 350 & 30 & 19 & 6 \\
\hline Science Direct & 1683 & 66 & 7 & 12 \\
\hline Bvs & 160 & 24 & 18 & 27 \\
\hline Total de artículos & 4697 & 152 & 48 & 5 \\
\hline
\end{tabular}

Para el control del riesgo de sesgo de selección, la investigadora, experta en el tema, contó con el apoyo de otros profesionales expertos en investigación, con el fin de dar validez a la elegibilidad de los artículos, basados en el objetivo del estudio y sin intereses particulares.

\section{Declaración de aspectos éticos}

Este artículo hace parte del proyecto de investigación "Construcción de un plan de cuidado de enfermería para el egreso hospitalario de adultos con heridas quirúrgicas abdominales de alta complejidad", y cuenta con aval del comité de ética de la Facultad de Enfermería de la Universidad Nacional de Colombia.

\section{RESULTADOS}

El total de estudios reportados en las bases de datos consultadas fue 4697. Al revisar los temas de acuerdo al objetivo del presente artículo, se extrajeron 27 que cumplieron con los criterios de inclusión. Todos ellos son cuantitativos.

El rango de autores por publicación es de dos a diecisiete, con un promedio de autores de siete. Del total de estudios que se incluyeron, el $44 \%$ son prospectivos, 25,9\% fueron publicados en el año 2013 , y $33,3 \%$ se produjeron en Japón. El idioma inglés fue prevalente con un $92,6 \%$. La mayoría de estos trabajos fueron realizados en el centro de cáncer gástrico del departamento de cirugía con
$55,6 \%$, y se observó predominio de la gastrectomía total con $45,9 \%$ (Tabla 2).

\section{Factor de impacto}

La temática de las revistas publicadas, en su mayoría, corresponde al área de medicina, con 15 publicaciones que integran las especialidades de gastroenterología, cirugía, oncología y anestesiología. Le siguen las áreas de enfermería, con dos publicaciones, y nutrición, políticas de salud y farmacología, con una publicación cada una.

Con respecto a los cuartiles, quince revistas se encontraban en categoría Q1 (Clinical Nutrition; Gastric Cancer; Plos One; Surgical Endose; World Journal of Gastroenterology; Annals of Surgery; World Journal of Surgery; Annals of Surgery Oncology; Journal Gastrointestinal Surgery; Journal Oncotarget; European Journal of Cancer; Trials; BMC Gastroenterology; Surgical Oncology; International Journal of Surgery). Cinco revistas estaban clasificadas en Q2 (Scandinavian Journal of Gastroenterology; Cancer Nursing; World Journal of Surgical Oncology; BMC Gastroenterology; Surgical Oncology), y cuatro en Q3 (Egyptian Journal of Anaesthesia; World Journal of Surgical Oncology; Annals of Medicina and Surgery; Asian Journal of Surgery). Por último, en el cuartil Q4 se clasificaban dos revistas: Revista Facultad Ciencias de La Salud y Revista Facultad de Medicina Universidad Nacional. 
Tabla 2. Resultados generales.

\begin{tabular}{|c|c|c|}
\hline Categoría & Subcategoría & Porcentaje (\%) \\
\hline \multirow[t]{9}{*}{ Años de publicación } & 2008 & 7,4 \\
\hline & 2011 & 7,4 \\
\hline & 2012 & 3,7 \\
\hline & 2013 & 25,9 \\
\hline & 2014 & 7,4 \\
\hline & 2015 & 14,8 \\
\hline & 2016 & 11,1 \\
\hline & 2017 & 7,4 \\
\hline & 2018 & 14,8 \\
\hline \multirow[t]{8}{*}{ País del estudio } & Japón & 33,3 \\
\hline & China & 18,5 \\
\hline & Corea del sur & 18,5 \\
\hline & Colombia & 7,4 \\
\hline & Italia & 7,4 \\
\hline & Reino Unido & 7,4 \\
\hline & Holanda & 3,7 \\
\hline & Egipto & 3,7 \\
\hline \multirow[t]{9}{*}{ Área } & Department of Surgery, centro de cáncer gástric. & 55,6 \\
\hline & Departamento de Cirugía Gastrointestinal, instituto de cáncer & 18,5 \\
\hline & Department of Hepatobiliary Surgery & 3,7 \\
\hline & Departamento de Medicina Interna & 3,7 \\
\hline & Instituto clínico de cirugía & 3,7 \\
\hline & Departamento de Medicina & 3,7 \\
\hline & Department of Anesthesia-Surgery & 3,7 \\
\hline & Departamento de Cirugía Digestiva & 3,7 \\
\hline & Departamento de Enfermería & 3,7 \\
\hline \multirow{7}{*}{$\begin{array}{l}\text { Tipo de } \\
\text { gastrectomía }\end{array}$} & Total & 45,9 \\
\hline & Subtotal & 18,9 \\
\hline & Laparoscopia & 13,5 \\
\hline & Abierta & 13,5 \\
\hline & Distal & 2,7 \\
\hline & Proximal & 2,7 \\
\hline & Preservación del píloro & 2,7 \\
\hline \multirow[t]{6}{*}{ Tipo de estudio } & Prospectivo & 44 \\
\hline & Retrospectivo & 24 \\
\hline & Ensayo clínico aleatorizado & 16 \\
\hline & Casos y controles & 8 \\
\hline & Transversal & 4 \\
\hline & Serie de caso & 4 \\
\hline
\end{tabular}


La mayor parte de las revistas que publicaron estos artículos son de Estados Unidos (6), seguidas por las de Reino Unido (5), Alemania (4), Países Bajos (3), Colombia (2), China (1), Egipto (1) y Taiwán (1).

\section{Clasificación Clavien-Dindo}

Los adultos con cáncer de estómago que han sido sometidos a una gastrectomía presentan en primera instancia complicaciones a nivel físico $\mathrm{y} / \mathrm{o}$ emocional, entre las que prevalecen, de acuerdo con la literatura, la infección órgano-espacio (9,1\%), el absceso intraabdominal (6,6\%), la infección de la herida o seroma $(5,6 \%)$, las fugas anastomóticas $(4,5 \%)$, las molestias gastrointestinales como vómito, mareo y náuseas $(3,3 \%)$, agotamiento $(3,1 \%)$ y alteración laboral $(3,1 \%)$, signos que podrían estar relacionados con depresión durante el posoperatorio. A continuación, se relacionan las complicaciones encontradas en la literatura de acuerdo con la clasificación de Clavien-Dindo por tipo de estudio.

Los ensayos clínicos controlados, según la clasificación Clavien-Dindo, reportan en el grado I que la complicación más frecuente está dada por las fugas anastomóticas $(19,9 \%)$. En el grado II surge la mayoría de complicaciones, siendo las más relevantes la infección de herida o seroma $(56,8 \%)$, el derrame pleural $(58,4 \%)$ y la neumonía $(28,3 \%)$. En el grado III, la complicación más frecuente está dada por la reoperación bajo anestesia general $(5,7 \%)$, principalmente por infección órgano-espacio e infección superficial. En el grado IV se revela una complicación de tipo respiratorio (8,5\%), y en el grado $\mathrm{V}$ aparece una mortalidad a este tipo de cirugía $(1,4 \%)$ (Figura 1).

De acuerdo a la clasificación Clavien-Dindo, los estudios de cohorte retrospectiva muestran solo tres grados de complicaciones, es decir, no presentan los dos últimos, que corresponden a complicaciones que amenazan la vida del paciente y requieren tratamiento en cuidados intermedios 0 intensivos y muerte de adultos por este tipo de cirugía.
En el grado I, la complicación principal está dada por el absceso intraabdominal (21,7\%); en el grado II se encuentra el mayor número de complicaciones, de las cuales la más frecuente es la infección de herida o seroma (6,8\%), y en el grado III la complicación más frecuente es la infección órgano-espacio (36,9\%) (Figura 2).

Los estudios de casos y controles presentan, al igual que los análisis retrospectivos, únicamente tres grados en la clasificación Clavien-Dindo. Se resaltan el absceso intraabdominal (20,3\%), la infección de herida o seroma $(15,6 \%)$ e íleo $(6,3 \%)$ en los grados I a III respectivamente (Figura 3 ).

Se reporta una serie de casos con cuatro complicaciones principales, correspondientes al 16,7\%: la atelectasia en el grado II de clasificación, la fístula abdominal, la parálisis intestinal y la reoperación bajo anestesia general por infección órgano-espacio en su mayoría e infección superficial.

Los estudios de cohortes prospectivas indican que existen complicaciones de grado I al III y V de la clasificación Clavien-Dindo. La más prevalente para el grado I es el síndrome de dumping (12,8\%); para el grado II, la diarrea infecciosa (12,1\%); para el grado III, el infarto cerebral (2,1\%) $y$, correspondiente al grado $\mathrm{V}$, una mortalidad de $2,1 \%$ (Figura 4).

Se reporta un exploratorio con cuatro complicaciones de igual magnitud, con un $25 \%$ cada una, las cuales son fugas anastomóticas y obstrucción intestinal para el grado $I$ de clasificación; infección de herida o seroma para el grado II, y dehiscencia de la herida para el grado III.

Un estudio transversal, de acuerdo a la clasificación Clavien-Dindo, únicamente reporta complicaciones grado I: molestias gastrointestinales (6,6\%), alteración laboral $(6,2 \%)$, agotamiento $(6,2 \%)$, debilidad (5,9\%), entre otras que están directamente relacionadas con el estado emocional y con una afectación socioeconómica (Figura 5). 


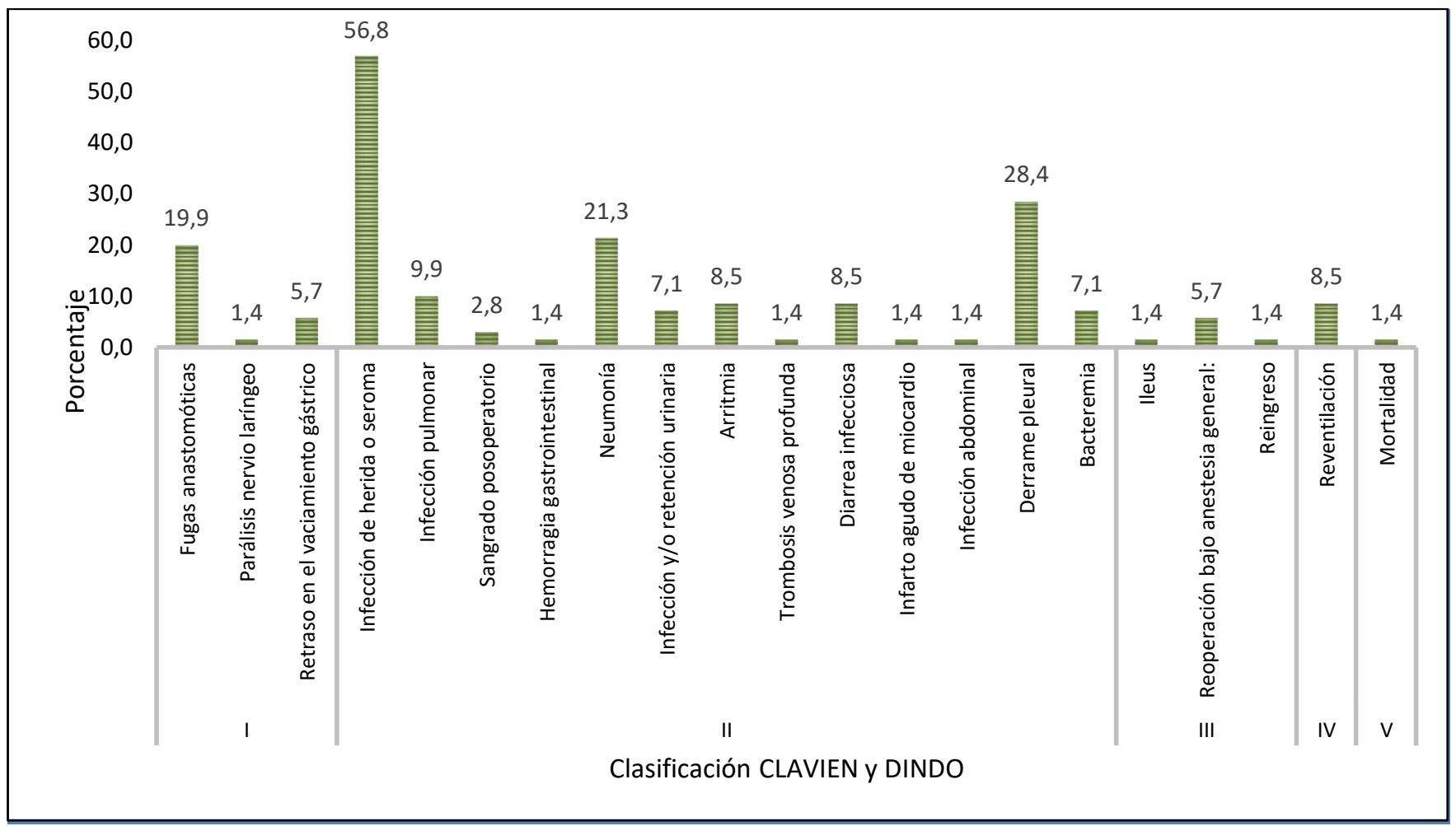

Figura 1. Complicaciones: ensayos clínicos controlados.

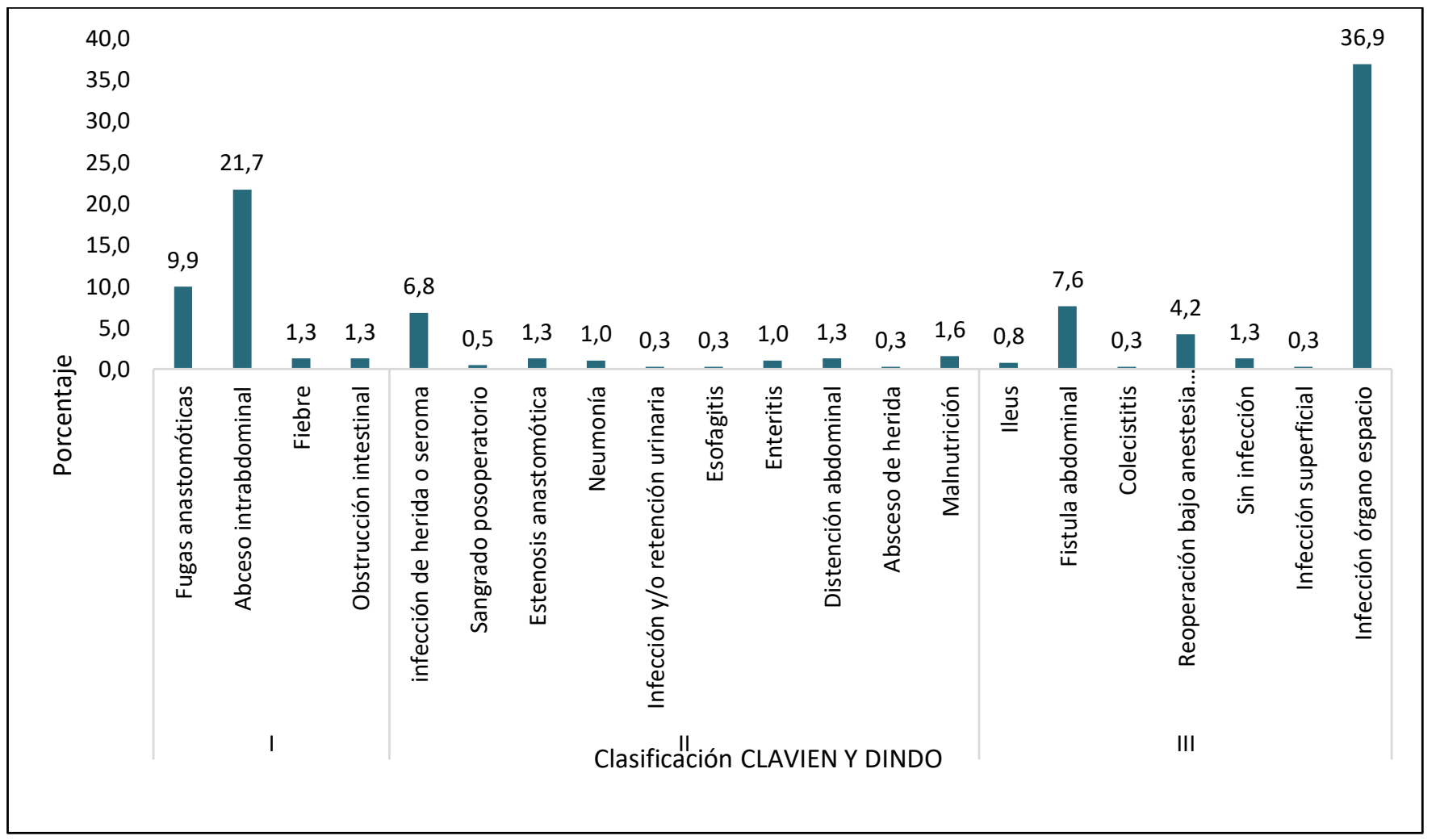

Figura 2. Complicaciones: estudios retrospectivos. 


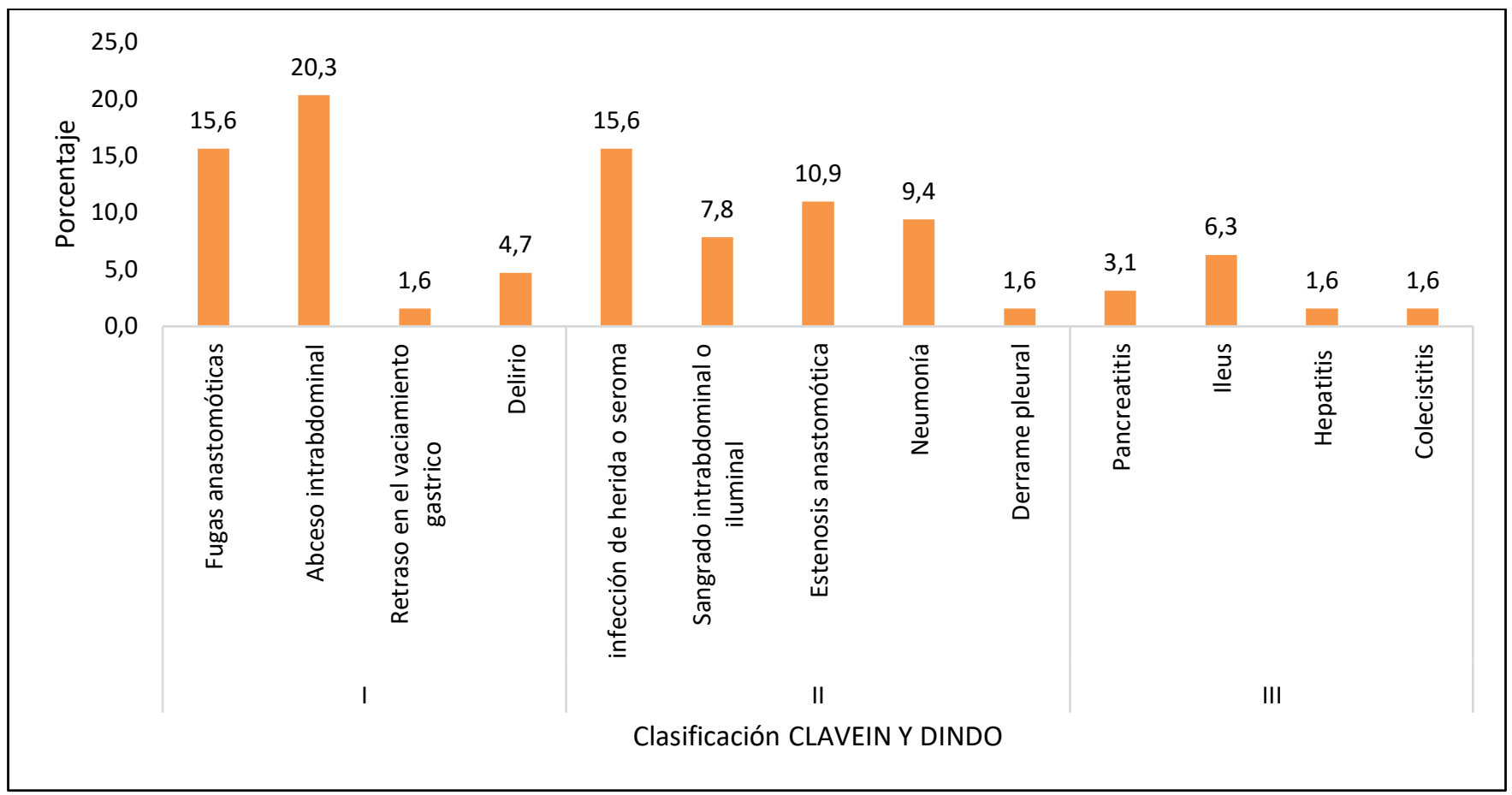

Figura 3. Complicaciones: estudios casos y controles.

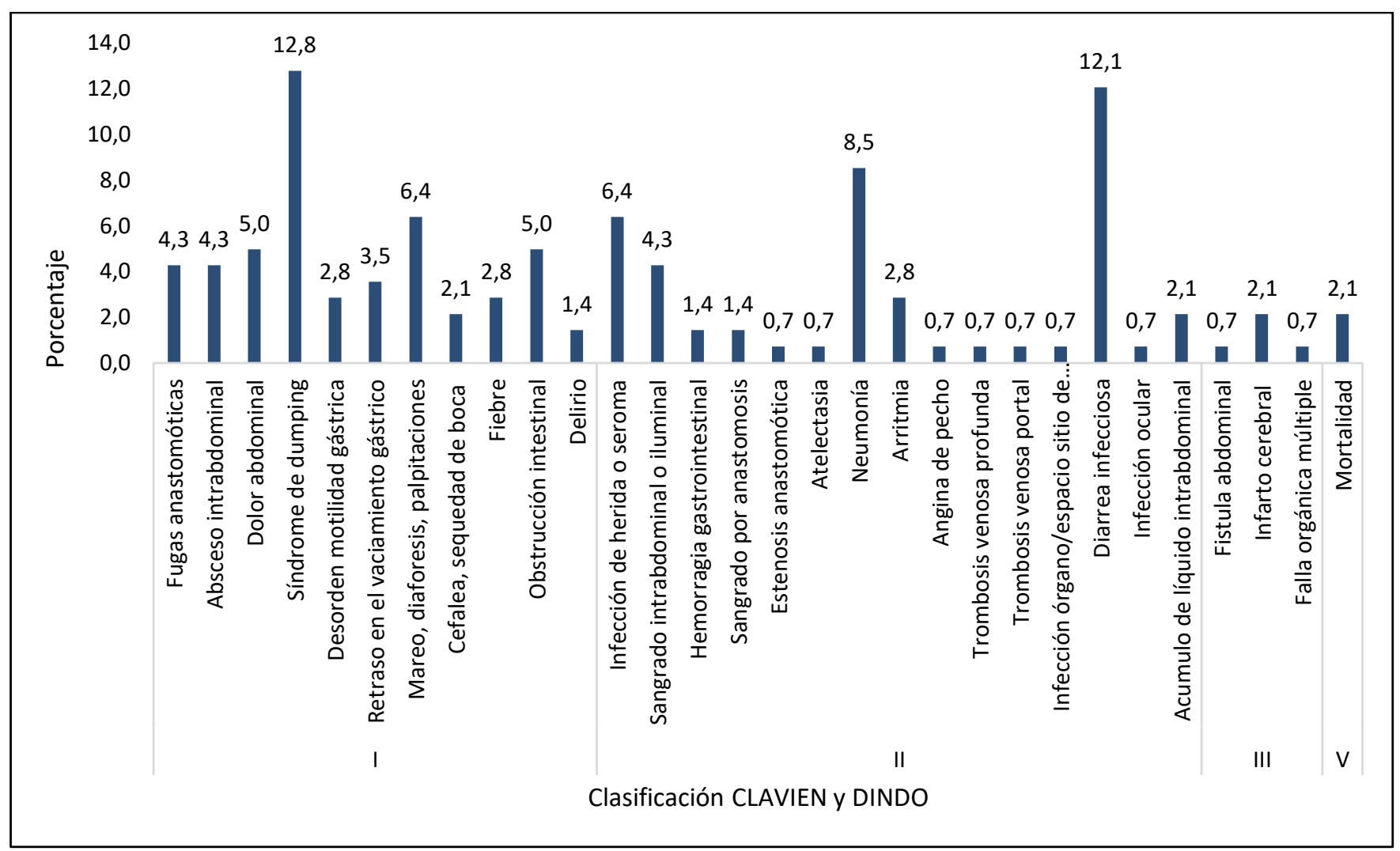

Figura 4. Complicaciones: estudios de cohortes prospectivas. 


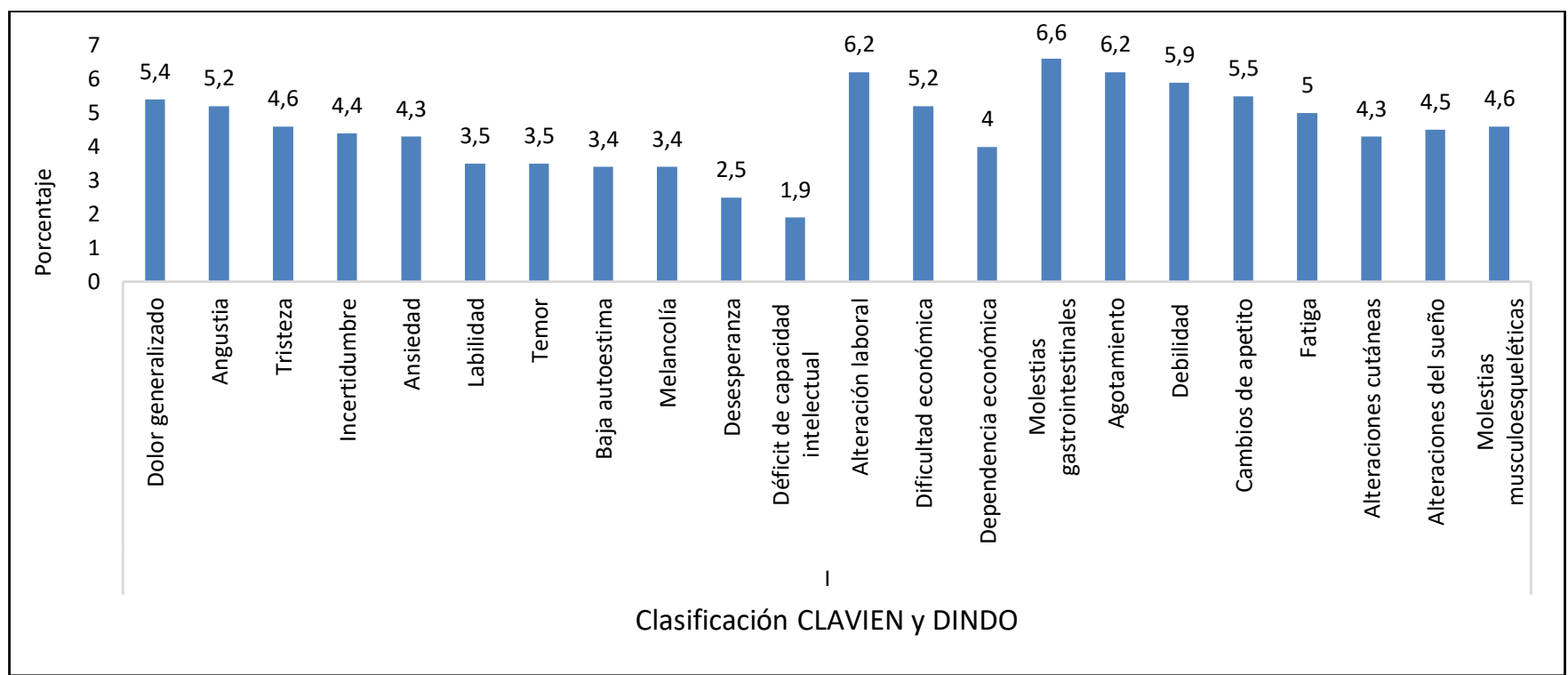

Figura 5. Complicaciones: estudios transversales.

\section{DISCUSIÓN}

Según la literatura, las complicaciones prevalentes encontradas luego de una gastrectomía en el adulto son infección de la herida o seroma, fugas anastomóticas ${ }^{21-30}$, infección órgano-espacio ${ }^{28,31}$, alteración laboral, molestias gastrointestinales y agotamiento $^{32}$. Las edades promedio de los pacientes participantes oscilan entre 58 y 62 años, y más del $60 \%$ del grupo total eran del sexo masculino ${ }^{33,34}$.

De acuerdo con los reportes, las infecciones posgastrectomía son ocasionadas por la herida quirúrgica, infección de vías urinarias y diarrea, dependiendo de la técnica quirúrgica empleada, siendo estas clasificadas como complicaciones menores $^{35}$. Las transfusiones, por otra parte, se asocian con presencia de infecciones del sitio quirúrgico ${ }^{31}$.

El sobrepeso ${ }^{36}$ constituye un factor de riesgo frente a la infección del sitio quirúrgico, la pérdida de sangre y tiempo quirúrgico, y las gastrectomías totales y distales. Además, cuanto mayor índice de masa corporal (IMC) tenga el paciente, mayor es la pérdida de peso. A su vez, la desnutrición también se convierte en un factor de riesgo ante complicaciones gastrointestinales como vaciamiento gástrico, fugas anastomóticas, hemorragia intraabdominal e infecciones posoperatorias ${ }^{28,37,38}$. No obstante, reportes indican que no existen diferencias estadísticamente significativas entre pacientes bien nutridos y mal nutridos. En estos últimos existe una incidencia de fugas anastomóticas ${ }^{27}$, y después de la gastrectomía algunos pacientes presentan dificultad para consumir nutrientes, un aspecto que se puede complicar por el tratamiento posterior, que es quimioterapia con radiación ${ }^{12}$.

El inicio de una alimentación temprana posoperatoria tiene como beneficios estancia hospitalaria corta, mejor calidad de vida, rentabilidad económica y el hecho de que no se aumenta el riesgo de complicaciones posoperatorias. Sin embargo, podrían presentarse fugas anastomóticas, infección del sitio quirúrgico, dehiscencia de la herida, íleo posoperatorio, neumonía, infección del tracto urinario, hemorragia intraabdominal, náuseas, vómito, diarrea y distención abdominal ${ }^{39}$. El alta hospitalaria temprana, por su parte, aumenta los reingresos por mala alimentación, estenosis anastomótica, obstrucción del intestino delgado y distención abdominal ${ }^{29,40}$.

La intolerancia a la alimentación representa una complicación común y un considerable desafío para la alimentación enteral temprana después de la cirugía por cáncer gástrico. Un estudio cuasiexperimental que abordó a dos grupos de 
pacientes sometidos a una gastrectomía, uno con una nutrición convencional y otro al que se le aplicó la técnica fast-tract, indica que la tasa general de complicaciones en este último grupo $(10,2 \%)$ fue significativamente menor que en el grupo convencional $(28,3 \%, p=0,019)$, en el cual se presentaron complicaciones como neumonía, infección por incisión, infección urinaria aumentada e infección abdominal, mientras que en el fast-track la neumonía y la infección por incisión fueron las más prevalentes ${ }^{22}$.

El resultado en la construcción de un modelo predictivo para la intolerancia a la alimentación por tubo en pacientes que son sometidos a una gastrectomía indica que se deben considerar como factores de riesgo independientes el historial preoperatorio de estreñimiento, el puntaje de ASA III, la puntuación de dolor posoperatorio de seis horas y un recuento alto de glóbulos blancos en el primer día después de la cirugía ${ }^{33}$. La mayor incidencia diaria de intolerancia desde el primer día posoperatorio hasta el tercero coincide con el periodo de estrés agudo, generando alteraciones hormonales y de absorción gastrointestinal ${ }^{41}$. En relación con esto, la literatura refiere que una persona sometida a gastrectomía total y que tenga sintomatología de anemia genera una mala absorción por deficiencia de hierro y vitamina C, lo que llevaría a molestias gastrointestinales y a una pérdida continua de peso ${ }^{42}$.

En los adultos, las fístulas de la anastomosis esófago-yeyunal, de acuerdo con la literatura, es una de las complicaciones más frecuentes, en 10$22 \%{ }^{43,44}$. Las fugas anastomóticas están asociadas con muerte, estancia hospitalaria alta, aumento de costos y disminución de la calidad de vida. Los factores de riesgo asociados a esta complicación se presentan por condiciones del paciente y por la técnica quirúrgica. En el primer caso se resaltan personas con edad mayor o igual a 65 años, hombres (4,2 veces mayor riesgo), hemoglobina menor o igual a $8 \mathrm{mg} / \mathrm{dl}$, antecedentes de malnutrición, enfermedad cardiovascular, insuficiencia pulmonar, diabetes, tabaquismo, falla renal y uso de esteroides. En cuanto al segundo aspecto, pueden influir un error en la técnica quirúrgica empleada, un tiempo quirúrgico prolongado y aumento de la tensión arterial ${ }^{42,45}$.

La gastrectomía por laparoscopia permite que la persona sienta menor dolor en el posoperatorio y una estancia hospitalaria más corta. Aun así, podrían presentarse complicaciones antes de cumplir 30 días del alta hospitalaria, como absceso abdominal, fístulas y fugas anastomóticas. Por otro lado, las gastrectomías abiertas generan mayor complicación de la herida, dando lugar a infección o seroma. No obstante, un estudio indica que no existe una diferencia significativa entre las complicaciones generadas por cirugía laparoscópica y las asociadas a cirugía abierta ${ }^{25}$.

Finalmente, un metaanálisis ${ }^{46}$ con personas asiáticas muestra que el uso de analgésicos es 1,73 veces menor, y el tiempo hasta el primer flato fue 0,51 días más con cirugía laparoscópica (LADG) que con cirugía abierta (ODG). Sin embargo, el tiempo hasta la primera ingesta de agua/alimento y la duración de la estancia posoperatorio no fueron significativos, así como la infección, la estenosis anastomótica, la fuga anastomótica, la hemorragia posoperatoria, la obstrucción posoperatoria/íleo, el vaciamiento gástrico retardado, el absceso intraabdominal y las complicaciones pancreáticas. La dehiscencia de la herida y el riesgo de complicaciones respiratorias fueron más bajos con LADG que con ODG, por lo que es más probable que un paciente con cáncer en estadio temprano sea sometido a una LADG debido a una mortalidad y seguridad oncológica similares, mejor seguridad quirúrgica, disminución de la morbilidad operatoria, menos trauma y recuperación acelerada. En cuanto a la gastrectomía distal y total, un estudio reporta que esta última se asocia con una mayor tasa de fuga anastomótica en comparación con la distal ${ }^{47-49}$.

\section{CONCLUSIÓN}

Las complicaciones de un adulto tratado por gastrectomía están relacionadas con diferentes factores de riesgo, unos asociados con comorbilidades de la persona y otros que tienen que ver con la técnica quirúrgica. Es decir, el preoperatorio desempeña un papel preponderante en el proceso quirúrgico dado que este incide en las 
complicaciones que se puedan presentar en el posoperatorio, especialmente en personas que tengan alteraciones nutricionales, ya sea por exceso o por déficit. Por lo tanto, se hace necesario construir un plan de cuidados interdisciplinario que visualice el proceso quirúrgico de manera transversal y promueva acciones que permitan controlar los factores de riesgo y las complicaciones posoperatorias que podrían ser prevenibles, así como disminuir la mortalidad por esta causa.

\section{DECLARACIÓN DE CONFLICTO DE INTERESES}

La autora manifiesta que durante la ejecución del trabajo o la redacción del manuscrito no han incidido intereses o valores distintos a los que usualmente tiene la investigación.

\section{REFERENCIAS BIBLIOGRÁFICAS}

1.Venerito $M$, Link A, Rokkas $T$, Malfertheiner P. Gastric cancer - clinical and epidemiological aspects. Helicobacter. 2016; 21(S1): 39-44. Doi: https://doi.org/10.1111/hel.12339.

2.Ang TL, Fock TL. Clinical epidemiology of gastric cancer. Singapur Med J. 2014; 55(12): 621-8. Doi: https://doi.org/10.11622/smedj.2014174.

3.Fock KM. The epidemiology and prevention of gastric cancer. Aliment Pharmacol \& Ther. 2014; 40(3): 250-60. Doi: https://doi.org/10.1111/apt.12814.

4.Morgan D. Early gastric cancer: epidemiology, clinical manifestations, diagnosis and staging [Internet]. UpToDate [citado 2018 Julio 24]. Disponible en: https://www.uptodate.com/contents/early-gastriccancer-epidemiologyclinical-manifestationsdiagnosis-and-staging.

5.Zhao G, Wu M, Wang X, Du Z, Zhang G. Effect of FABP5 gene silencing on the proliferation, apoptosis and invasion of human gastric SGC-7901 cancer cells. Oncology Letters. 2017; 14(4): 772-8. Doi: https://doi.org/10.3892/ol.2017.6748.
6.Rugge M, Genta RM, Di Mario F, El-Omar EM, ElSerag HB, Fassan $M$, et al. Gastric Cancer as Preventable Disease. Clinical Gastroenterology and Hepatology. 2017; 15(12): 1833-43. Doi: https://doi.org/10.1016/j.cgh.2017.05.023.

7.Oh SJ, Choi WB, Song J, Hyung WJ, Choi SH, Noh $\mathrm{SH}$, et al. Complications Requiring Reoperation after Gastrectomy for Gastric Cancer: 17 Years Experience in a Single Institute. J Gastrointest Surg. 2008; 13(2009): 239-45. Doi: https://doi.org/10.1007/s11605-008-0716-3.

8.Instituto Nacional de Cancerología. Análisis de situación del cáncer en Colombia 2015 [Internet]. Bogotá, Colombia: Instituto Nacional de Cancerología; 2017 [citado 2019 Enero 20]. Disponible en: https://www.cancer.gov.co/files/libros/archivos/Sit uacion.

9.Ospina ML, Huertas JA, Montaño JI, Rivillas JC. Observatorio Nacional de Cáncer Colombia. Facultad Nacional de Salud Pública: El escenario para la salud pública desde la ciencia. 2015; 33(2): 262-276. Disponible en: https://dialnet.unirioja.es/servlet/articulo?codigo= 5107319

10.Caselli B, Cabrera J, Molina H. Análisis de supervivencia en gastrectomía subtotal por cáncer gástrico. Concepción, Chile. CIMEL Ciencia e Investigación Médica Estudiantil Latinoamericana. 2006; 11(2): 56-61. Disponible en: https://www.redalyc.org/pdf/717/71711204.pdf.

11. Henao FJ, Rojas OA, Mejía AF. Extensión de la linfadenectomía para el tratamiento quirúrgico del adenocarcinoma gástrico en el Hospital Universitario San Ignacio: cohorte prospectiva con análisis de supervivencia. Rev Colomb Cir. 2001; 16(2): 65-71. Disponible en: https://pesquisa.bvsalud.org/portal/resource/pt/lil $-325775$.

12.American Cancer Society. Cirugía para el cáncer de estómago [Internet]. 2017. Disponible en: https://www.cancer.org/es/cancer/cancer-deestomago/tratamiento/tipos-de-cirugias.html. 
13.Eulufi FC, Véliz MM. Manual de patología quirúrgica. Ediciones UC: Pontificia Universidad Católica de Chile; 2021. Disponible en: https://n9.cl/097p

14. Merkow RP, Ju MH, Chung JW, Hall BL, Cohen $\mathrm{ME}$, Williams MV, et al. Underlying Reasons Associated With Hospital Readmission Following Surgery in the United States. JAMA. 2015; 313(5): 483-95.

Doi: https://doi.org/10.1001/jama.2014.18614.

15.Selby LV, Vertosick AE, Sjoberg DD, Schattner MA, Janjigian YY, Brennan MF, et al. Morbidity after Total Gastrectomy: Analysis of 238 Patients. J Am Coll Surg. 2015; 220(5): 863-71. Doi: https://doi.org/10.1016/j.jamcollsurg.2015.01.058.

16.Domínguez Carrera JM. Factores asociados a morbi-mortalidad post-gastrectomía laparoscópica por cáncer gástrico [tesis doctoral]. Universidad de Vigo, Departamento de Bioquímica, Genética e Inmunología; $2015 . \quad$ Disponible en: http://www.investigo.biblioteca.uvigo.es/xmlui/bit stream/handle/11093/634/Factores_asociados_a_ morbi-mortalidad.pdf?sequence $=1$.

17.Peña LL, Reyes TT. Efectividad de la gastrectomía laparoscópica en pacientes con cáncer gástrico [trabajo académico para optar el título de especialidad en enfermería centro quirúrgico]. Lima, Perú: Universidad Privada Norbert Wiener, Facultad de Ciencias de la Salud; 2018. Disponible en: http://repositorio.uwiener.edu.pe/handle/1234567 $89 / 2672$.

18.Vandenbroucke JP, Von EE, Altman DG, Gøtzsche PC, Mulrow CD, Pocock SJ, et al. Mejorar la comunicación de estudios observacionales en epidemiología (STROBE): explicación y elaboración. Gac Sanit. 2009; 23(2): 158e1-158e28. Disponible en:

http://scielo.isciii.es/scielo.php?script=sci_arttext\& pid=S0213-91112009000200015\&lng=es.

19.Caravia Pubillones I, Vela Carabia I. Aplicabilidad de la clasificación de Clavien y Dindo en las complicaciones quirúrgicas urológicas. Revista Cubana de Urología. 2015; 4(2). Disponible en: http://www.revurologia.sld.cu/index.php/rcu/articl e/view/245/251.

20.Clavien P, Barkun J, De Oliveira M, Vauthey J, Dindo $D$, Schulick $R$, et al. La clasificación de complicaciones quirúrgicas de Clavien-Dindo: experiencia de cinco años. Anales de Cirugía. 2009; 250(2): 187-96. Doi: https://doi.org/10.1097/SLA.0b013e3181b13ca2.

21.Jing-Xiang $S$, Xiao-Huang $T$, Bing W, Chen L, Zaizhong $\mathrm{Z}$, Li-ying $\mathrm{L}$, et al. "Fast track" rehabilitation after gastric cancer resection: experience with 80 consecutive cases. BMC Gastroenterol. 2014, 14(2014): 147. Doi: https://doi.org/10.1186/1471230X-14-147.

22.Fan F, Gang J, Ji-Peng L, Xiao-Hua L, Hai S, ZhengWei $Z$, et al. Fast-track surgery could improve postoperative recovery in radical total gastrectomy patients. World J Gastroenterol. 2013; 19(23): 364248. Doi: https://doi.org/10.3748/wjg.v19.i23.3642.

23.Bowrey DJ, Baker M, Halliday V, Thomas AL, Pulikottil-Jacob R, Smith $K$, et al. A randomised controlled trial of six weeks of home enteral nutrition versus standard care after oesophagectomy or total gastrectomy for cancer: report on a pilot and feasibility study. Bio Med Central. 2015; 16(2015): 531. Doi: https://doi.org/10.1186/s13063-015-1053-y.

24.Barlow R, Price P, Reid T, Hunt S, Clark GW, Havard $T$, et al. Prospective multicentre randomised controlled trial of early enteral nutrition for patients undergoing major upper gastrointestinal surgical resectionq. Clinical Nutrition. 2011; 30(5): 560-6. Doi: https://doi.org/10.1016/j.clnu.2011.02.006.

25.Lee MS, Lee JH, Park DJ, Lee HJ, Kim HH, Yang HK. Comparison of short- and long-term outcomes of laparoscopicassisted total gastrectomy and open total gastrectomy in gastric cancer patients. Surg Endosc. 2013; 27(2013): 2598-605. Doi: https://doi.org/10.1007/s00464-013-2796-8.

26.Li QG, Li P, Tang D, Chen J, Wang DR. Impact of postoperative complications on long-term survival after radical resection for gastric cancer. World J 
Gastroenterol 2013; 19(25): 4060-5. Doi: https://doi.org/10.3748/wjg.v19.i25.4060.

27.Pacelli F, Bossola M, Rosa F, Tortorelli AP, Papa V, Doglietto GB. Is malnutrition still a risk factor of postoperative complications in gastric cancer surgery? Clin Nutr. 2008; 27(3): 398-407. Doi: https://doi.org/10.1016/j.clnu.2008.03.002.

28.Fukuda Y, Yamamoto K, Hirao M, Nishikawa K, Maeda S, Haraguchi $N$, et al. Prevalence of Malnutrition Among Gastric Cancer Patients Undergoing Gastrectomy and Optimal Preoperative Nutritional Support for Preventing Surgical Site Infections. Ann Surg Oncol. 2015; 22(2015): S77885. Doi: https://doi.org/10.1245/s10434-015-48209.

29. Honda M, Hiki N, Nunobe S, Ohashi M, Mine S, Watanabe $M$, et al. Unplanned admission after gastrectomy as a consequence of fast-track surgery: a comparative risk analysis. Gastric Cancer. 2016 Jul; 19(2016): 1002-7. Doi: https://doi.org/10.1007/s10120-015-0553-5.

30.Segami K, Aoyama T, Kano K, Maezawa Y, Nakajima T, Ikeda K, et al. Risk factors for severe weight loss at 1 month after gastrectomy for gastric cancer. Asian J Surg. 2018; 41(4): 349-55. Doi: https://doi.org/10.1016/j.asjsur.2017.02.005.

31.Migita K, Takayama T, Matsumoto S, Wakatsuki $\mathrm{K}$, Enomoto K, Tanaka, et al. Risk Factors for Surgical Site Infections After Elective Gastrectomy. J Gastrointest Surg. 2012; 16(2012): 1107-15. Doi: https://doi.org/10.1007/s11605-012-1838-1.

32. Carrillo GM, Bayona HA, Arias EM. Perfil y carga de la enfermedad de personas con cáncer gástrico sometidas a gastrectomía. Rev Fac Med. 2018; 66(1): 13-8. Doi: https://doi.org/10.15446/revfacmed.v66n1.60273.

33.Xiaoyong $W$, Xuzhao L, Deliang $Y$, Pengfei $Y$, Zhenning $H$, Bin $B$, et al. Construction of a model predicting the risk of tube feeding intolerance after gastrectomy for gastric cancer based on 225 cases from a single Chinese center. Oncotarget. 2017; 8(59): $\quad 99940-9 . \quad$ Doi: https://doi.org/10.18632/oncotarget.21966.
34.Martínez JP, González CE, Cristancho L, Arroyave Y, Calvache JA. Gastrectomía total por cáncer gástrico en el hospital universitario San José de Popayán. Cohorte retrospectiva de 16 años. Revista Facultad Ciencias de la Salud. Universidad del Cauca. 2013; 15(4): 25-31. Doi: https://revistas.unicauca.edu.co/index.php/rfcs/art icle/view/42/41.

35.Grantcharov TP, Kehlet $\mathrm{H}$. Laparoscopic gastric surgery in an enhanced recovery programme. $\mathrm{Br} \mathrm{J}$ Surg. 2010 Oct; 97(10): 1547-51. Doi: https://doi.org/10.1002/bjs.7184.

36. Hirao M, Tsujinaka $\mathrm{T}$, Imamura $\mathrm{H}$, Kurokawa $\mathrm{Y}$, Inoue K, Kimura $Y$, et al. Osaka Gastrointestinal Cancer Chemotherapy Study Group (OGSG). Overweight is a risk factor for surgical site infection following distal gastrectomy for gastric cancer. Gastric Cancer. 2013 Apr; 16(2013): 239-44. Doi: https://doi.org/10.1007/s10120-012-0174-1.

37.Zhuang CL, Wang SL, Huang DD, Pang WY, Lou N, Chen BC, et al. Risk Factors for Hospital Readmission after Radical Gastrectomy for Gastric Cancer: A Prospective Study. PLoS One. 2015 Apr 27; 10(4): e0125572.

https://doi.org/10.1371/journal.pone.0125572.

38. Kim H, Suh EE, Lee HJ, Yang HK. The Effects of Patient Participation-Based Dietary Intervention on Nutritional and Functional Status for Patients With Gastrectomy: A Randomized Controlled Trial. Cancer Nurs. 2014 Mar-Apr; 37(2): E10-20. Doi: https://doi.org/10.1097/NCC.0b013e31829193c8.

39.Tweed $\mathrm{T}$, Van Eijden $\mathrm{Y}$, Tegels J, Brenkman $\mathrm{H}$, Ruurda J, Van Hillegersberg R, et al. Safety and efficacy of early oral feeding for enhanced recovery following gastrectomy for gastric cancer: A systematic review. Surg Oncol. 2019 Mar; 28: 88-95. Doi: https://doi.org/10.1016/j.suronc.2018.11.017.

40.Acher AW, Campbell-Flohr SA, Brenny-Fitzpatrick $M$, Leahy-Gross KM, Fernandes-Taylor S, Fisher AV, et al. Improving Patient-Centered Transitional Care after Complex Abdominal Surgery. J Am Coll Surg. 2017 Aug; 225(2): 259-65. Doi: https://doi.org/10.1016/j.jamcollsurg.2017.04.008. 
41. Warren J, Bhalla V, Cresci G. Postoperative Diet Advancement: Surgical Dogma vs Evidence-Based Medicine. Nutr Clin Pract. 2011 Apr; 26(2): 115-25. Doi: https://doi.org/10.1177/0884533611400231.

42.Gong W, Li J. Combat with esophagojejunal anastomotic leakage after total gastrectomy for gastric cancer: A critical review of the literature. Int J Surg. 2017 Nov; 47: 18-24. Doi: https://doi.org/10.1016/j.ijsu.2017.09.019.

43.Andreollo NA, Lopes LR, Coelho JDS. Postoperative complications after total gastrectomy in the gastric cancer. Analysis of 300 patients. Arq Bras Cir Dig. 2011; 24(2): 126-130. Doi: https://doi.org/10.1590/S010267202011000200007.

44.Inokuchi $M$, Otsuki S, Fujimori $\mathrm{Y}$, Sato $\mathrm{Y}$, Nakagawa M, Kojima K. Systematic review of anastomotic complications of esophagojejunostomy after laparoscopic total gastrectomy. World J Gastroenterol. 2015 Aug 28;21(32):9656-65. Doi: https://doi.org/10.3748/wjg.v21.i32.9656.

45.Lee KG, Lee HJ, Yang JY. Risk factors associated with complication following gastrectomy for gastric cancer: retrospective analysis of prospectively collected data based on the Clavien-Dindo system. J Gastrointest Surg. 2014; 18(7): 1269-77. Doi: 10.1007/s11605-014-2525-1.

46.Zhang CD, Yamashita $H$, Zhang $S$, Seto $Y$, Reevaluation of laparoscopic versus open distal gastrectomy for early gastric cancer in Asia: A metaanalysis of randomized controlled trials. Int J Surg. 2018; 56: 31-43. Doi: https://doi.org/10.1016/j.ijsu.2018.05.733.

47.Zhengyan L, Bin B, Fengni X, Qingchuan Z. Distal versus total gastrectomy for middle and lower-third gastric cancer: A systematic review and metaanalysis. Int J Surg. 2018; 53: 163-70.Doi: https://doi.org/10.1016/j.ijsu.2018.03.047.

48.Lee JH, Park DJ, Kim HH, Lee HJ, Yang HK. Comparison of complications after laparoscopyassisted distal gastrectomy and open distal gastrectomy for gastric cancer using the ClavienDindo classification. Surg Endosc. 2011; 26(2012), 1287-95. Doi: https://doi.org/10.1007/s00464-0112027-0.

49. Kim DJ, Lee JH, Kim W. Comparison of the major postoperative complications between laparoscopic distal and total gastrectomies for gastric cancer using Clavien-Dindo classification. Surg Endosc. 2015; 29(2015): 3196-204. Doi: https://doi.org/10.1007/s00464-014-4053-1. 